\title{
Thiophenol detection using an AIE fluorescent probe through self- assembly with TPE-based glycoclusters
}

Received 00th January 20xx, Accepted 00th January 20xx

DOI: $10.1039 / x 0 x \times 00000 x$

\author{
Lei Dong, ${ }^{a}$ Guo-Rong Chen, ${ }^{b}$, Xiao-Peng $\mathrm{He}{ }^{* \mathrm{~b}}$ and Sébastien Vidal*a
}

We describe a novel green-emitting tetraphenylethylene-dicyanomethylene-4H-pyran (TPE-DCM) based fluorescent probe (TD-1). Conjugating TPE and DCM moieties allowed TD-1 to display high selectivity for thiophenol with excellent AIE properties in aqueous solution. Nevertheless, the poor water-solubility from the hydrophobic structure resulted in the weak and unstable emission intensity. Non-covalent self-assembly of TD-1 with TPE glycocluster (TPE2S) led to a largely improved water solubility producing a reliable and stable sensing system. The corresponding glyco-probe could detect sensitively exogenous thiophenol concentrations in PBS buffer or environmental water samples.

\section{Introduction}

Thiophenol ( $\mathrm{PhSH})$, as an important organosulfur compound, is the simplest aromatic thiol and essential starting materials extensively applied in organic synthesis of agrochemicals, pharmaceuticals and dyes. ${ }^{1-3}$ Different from aliphatic thiols including cysteine (Cys), homocysteine (Hcy) and glutathione (GSH), which play important roles in biological systems, ${ }^{4}$ thiophenol and its derivatives are pollutants with high toxicity for the environment. The toxicity of thiophenol is high in fish and mouse with a median lethal dose (LC50) from 0.01 to 0.04 $\mathrm{mM}$ or as low as $46.2 \mathrm{mg} / \mathrm{kg} .{ }^{5,} 6$ Long-term exposure to thiophenol liquid or vapor in water or soil is detrimental to human health by triggering series of severe systemic injuries, such as damage to the central nervous system, muscle weakness and even death. ${ }^{7}$ Therefore, a convenient, rapid, accurate, sensitive and selective method is necessary for the detection of thiophenol pollution in the environment.

Compared with traditional methods such as HPLC, UV-vis and chromatography-mass analysis, ${ }^{8,9}$ fluorescence spectroscopy is widely applied for thiophenol detection due to its high sensitivity, simple processing and low detection limits. A reactive moiety is typically introduced to a fluorophore scaffold to quench its fluorescence, and then a reaction with thiophenol triggers a cascade that would release the moiety enhancing fluorescence. 2,4-Dinitrobenzene was extensively used as the

\footnotetext{
a. Institut de Chimie et Biochimie Moléculaires et Supramoléculaires, Laboratoire de Chimie Organique 2-Glycochimie, UMR 5246, CNRS and Université Claude Bernard Lyon 1, Université de Lyon, 1 Rue Victor Grignard, F-69622 Villeurbanne, France. Email: sebastien.vidal@univ-lyon1.fr

b. Key Laboratory for Advanced Materials and Joint International Research Laboratory of Precision Chemistry and Molecular Engineering, Feringa Nobel Prize Scientist Joint Research Center, School of Chemistry and Molecular Engineering, East China University of Science and Technology, 130 Meilong Rd., Shangha 200237, PR China. Email:xphe@ecust.edu.cn

† Footnotes relating to the title and/or authors should appear here.

Electronic Supplementary Information (ESI) available: [details of any supplementary information available should be included here]. See DOI: 10.1039/x0xx00000x
}

reactive group since when this group is conjugated through sulfonyl ether, ${ }^{4,10-12}$ sulphonamide ${ }^{13-16}$ and sulfonate ${ }^{17-19}$ bonds to a fluorophore could lead to the production of fluorogenic probes for thiophenol selectively over other aliphatic thiols. ${ }^{20}$ The electron-deficient 2,4-dinitrobenzene supresses the fluorescence emission of organic dyes by the intramolecular charges transfer (ICT) ${ }^{21,22}$ or photo-induced electronic transfer (PET) mechanism. ${ }^{13}, 18,23$ Recently, fluorescent probes for thiophenol detection have been developed with high sensitivity, selectivity and short response time. For example, a squarainebased NIR probe for the detection of thiophenol through colorimetric and "off-on" fluorometric response with high watersolubility was developed through conjugation with glucose. ${ }^{19} \mathrm{~A}$ dicyanomethylene-4H-pyran (DCM) based probe with a large Stokes shift $(159 \mathrm{~nm})$ and low detection limit $(8.3 \mathrm{nM})$ was also constructed. ${ }^{24}$ However, most fluorescent probes exhibited low reactivity and fluorescence intensity due to their poor watersolubility and the notorious ACQ (aggregation-induced quenching) effect.

Aggregated induced emission (AIE) is widely used in organic fluorescent probe design to overcome ACQ in aqueous solution ${ }^{25-27}$ based on the pioneering studies of Prof Ben Zhong Tang who coined the concept of AIE in 2001.28 Tetraphenylethene (TPE) has attracted much attention due to its readily available structural modification and functionalization as well as its AIE properties and photostability. ${ }^{29-31}$ However, the wavelength of the blue emission largely overlaps the autoluminescence of biomolecules. As a consequence, fluorophores with a longer emission wavelength such as dicyanomethylene$4 H$-pyran (DCM) have been applied for the design of fluorescent probes. $^{32-34}$

We described herein the conjugation through a $\mathrm{C}=\mathrm{C}$ double bond between the DCM and TPE fluorophores to obtain a novel TPE-DCM fluorophore with yellow fluorescence emission as well as AIE properties for the detection of thiophenol. After functionalizing the electron-poor 2,4-dinitrobenzene on the fluorophore, restricted AIE effect and blocked ICT effect were 
observed to "turn-off" the fluorescence in aqueous solution. However, the poor water-solubility from the hydrophobic structure of TD-1 led to weak and unstable fluorescence emission in aqueous solution, due to precipitation of probes from the solution. Tetraphenylethene (TPE) based glycoclusters (TPE2S) have an outstanding ability to improve the water- solubility, fluorescence stability and sensitivity of TD-1 for thiophenol detection after self-assembly (Scheme 1). Therefore, the self-assembled glyco-probe was explored to quantify the concentration of thiophenol in real water samples (Rhône River and Saône River, Lyon).

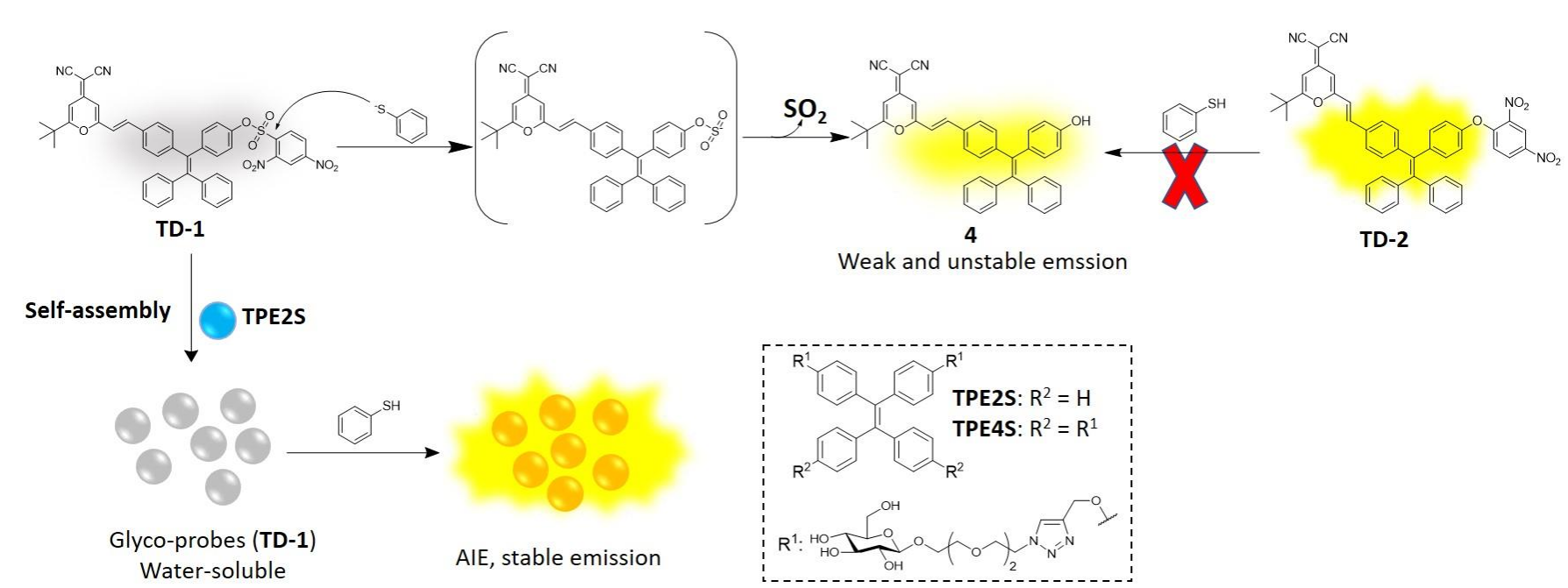

Scheme 1 TPE-DCM based fluorescent probe (TD-1) and its glycol-probes self-assembled with TPE-based glycoclusters (TPE2S) for thiophenol (PhSH) detection.

\section{Results and discussion}

\section{Chemical synthesis}

The synthesis procedures of novel TPE-DCM based fluorescent probe and TPE-based glycoclusters were shown (Scheme S1, ESI $\dagger$ ). The synthesis of the conjugate started from the known TPE-aldehyde $\mathbf{1}^{35}$ and DCM analogue $\mathbf{2}^{36}$ through an aldolisation/crotonization sequence to afford TPE-DCM $\mathbf{3}$. Demethylation of the phenol group with $\mathrm{BBr}_{3}$ produced the fluorescent probe 4. 2,4-Dinitrobenzene was functionalized on the phenol 4 to obtain two fluorescent probes TD-1 and TD-2 with different molecular architectures. TPE-based glycoclusters functionalized by two monosaccharides (TPE2S) was synthesized from dipropargyl TPE compound S5. Through CuAAC cycloaddition, acetyl protected azide-glucose were conjugated on the TPE core. Then TPE-based glycoclusters 5 was deprotected in aqueous $\mathrm{MeOH}$ solution with $\mathrm{Et}_{3} \mathrm{~N}$ to obtain the glycocluster TPE2S. TPE-based glycoclusters (TPE4S) was synthesized similarly according to the literature. ${ }^{37}$

\section{Response mechanism}

The performance of two probes for thiophenol detection was evaluated. The fluorescence of TD-2 remained unquenched and displayed only a limited fluorescence emission decrease after incubation with thiophenol (Fig. S1, ESI $\dagger$ ). In contrast, TD-1 displayed a much weaker initial fluorescence emission $(\Phi=$ $0.003)$, and an obvious fluorescence enhancement at $570 \mathrm{~nm}(\Phi$ $\left.=0.083, \lambda_{\mathrm{ex}}=480 \mathrm{~nm}\right)$ was observed probably through recovering of the ICT after reaction with thiophenol, along with a slight red-shift of the absorption peak (Fig. S1, ESI $\uparrow$ ). The mechanism of fluorescence enhancement was proposed as follows: the negative thiophenol $\left(\mathrm{PhS}^{-}\right)$ion was nucleophilic enough to add to the highly electrophilic ipso-carbon atom of the 2,4-dinitrophenyl group. Cleavage of the S-O bond and release of $\mathrm{SO}_{2}$ generated the phenol derivative 4 (Scheme 1). Mass spectrometry analysis indicated that TD-1 after responding to thiophenol generated phenol derivative $4(\mathrm{~m} / \mathrm{z}, 573.3)$. On the opposite, incubation of TD-2 with thiophenol did not produce phenol $\mathbf{4}$ and hence this compound was not further studied herein (Fig. S2a, ESI $\dagger$ ). To further prove 2,4-dinitrobenzene was the leaving group, HPLC analysis was performed to identify TD-1, 4 and thiophenol. After incubating a sub-stoichiometric amount of thiophenol with the probe, the corresponding peaks of TD-1 and 4 were observed. Then TD-1 was totally converted into compound 4 when using excessive thiophenol (Fig. S2b, ESI $\dagger$ ).

\section{Spectral properties of TD-1}

The sensing performances of TD-1 $(10 \mu \mathrm{M})$ for thiophenol in a phosphate buffered saline (PBS) solution (10 mM with $10 \% \mathrm{THF})$ were measured. The fluorescence enhancement of TD-1 was measured after addition of an excess of thiophenol at different $\mathrm{pH}$. We found that the fluorescence intensity kept increasing at $\mathrm{pH}$ 3-7 and reached equilibrium above $\mathrm{pH} 7$, which suggests that the thiophenate $\left(\mathrm{PhS}^{-}\right)$required at higher $\mathrm{pH}$ values to activate fluorescence. As a result, the probe TD-1 is capable of sensing thiophenol in neutral or basic solutions (Fig. 1a).

Then, we measured the AIE properties of TD-1 after reaction with thiophenol $(50 \mu \mathrm{M})$ in PBS solution ( $\mathrm{pH} 7.3)$ with different ratio of THF. The stronger emission of TD-1 in 50\% PBS solution indicated that 2,4-dinitrobenzene could not quench the fluorescence completely probably due to the electron rich moieties on the TPE moiety. The weakening of fluorescence 
intensity for TD-1 upon addition of PBS into THF was caused by the ACQ effect. We speculate that the bulky 2,4dinitrobenzene group restricted the rotation of TPE-DCM, hence blocking the AIE effect. Thereby, the fluorescence of TD-1 was switched-off by ICT effect and ACQ effect in aqueous buffer. The fluorescence emission was then largely enhanced by AIE effect after incubating with thiophenol (leading to fluorophore 4) when increasing PBS ratio from $50 \%$ to $80 \%$ (Fig. 1b). The phenol 4 recovered the AIE properties hence displayed enhanced emission intensity. Further addition of PBS buffer led to a decrease of fluorescence emission, suggesting an insufficient water-solubility of the probe to function in a pure aqueous medium. We supposed that aggregation of fluorophore $\mathbf{4}$ resulted in precipitation and a strong decrease of soluble material leading to a decreased signal.
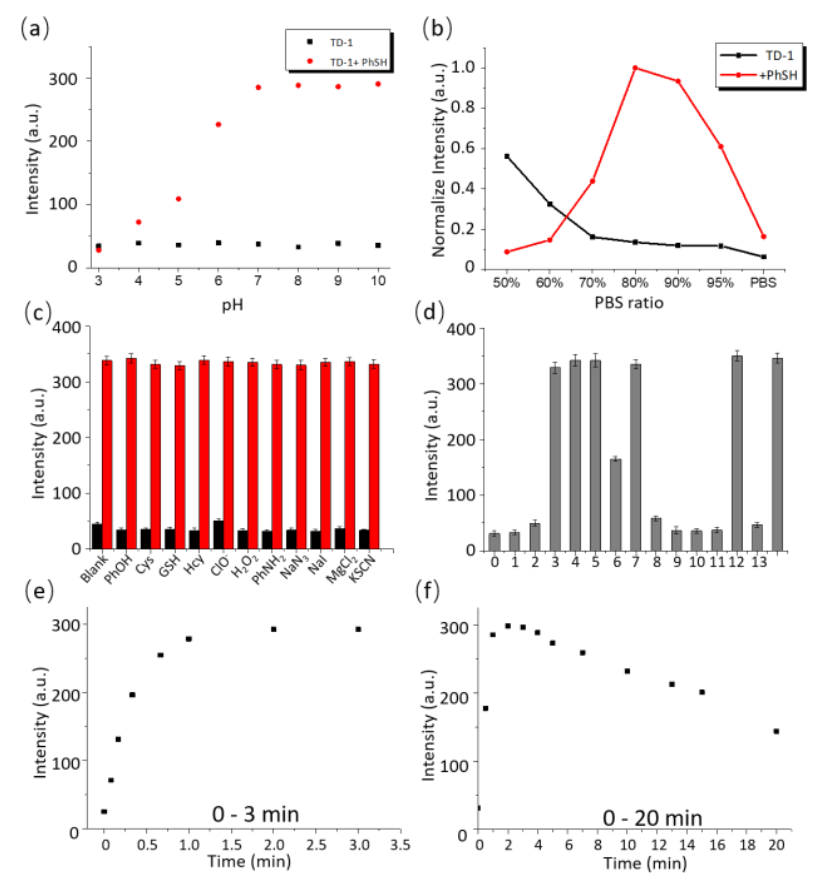

Figure 1 (a) Fluorescence variations of TD-1 $(10 \mu \mathrm{M})$ before and after responding to thiophenol $(100 \mu \mathrm{M})$ during 3 min in PBS buffer $(\mathrm{pH} 7.3)$ with $10 \%$ THF ( $\mathrm{pH}$ was adjusted with $\mathrm{HCl} 0.6 \mathrm{M}$ or $\mathrm{NaOH} 2 \mathrm{M}$ ). (b) $\mathrm{ACQ}$ and AIE effect influenced TD-1 response to thiophenol $(100 \mu \mathrm{M})$ with addition of PBS buffer ( $\mathrm{pH}$ 7.3) in THF during $10 \mathrm{~min}$. (c) The fluorescence variation of TD-1 $(10 \mu \mathrm{M})$ response to potential interfering species $(50 \mu \mathrm{M})$ without (black bar) or with (red bar) thiophenol $(100 \mu \mathrm{M})$ during 3 min. (d) TD-1 (10 $\mu \mathrm{M})$ response to aliphatic and aromatic thiols $(100 \mu \mathrm{M})$ during 3 min, Reagents: (0) Blank, (1) 1,2-ethanedithiol, (3) cyclohexanethiol, (4) $m-\mathrm{NH}_{2} \mathrm{C}_{6} \mathrm{H}_{4} \mathrm{SH}$, (5) $p-\mathrm{NH}_{2} \mathrm{C}_{6} \mathrm{H}_{4} \mathrm{SH}$ ， (5) $p$ $\mathrm{MeOC}_{6} \mathrm{H}_{4} \mathrm{SH}$, (6) $p-\mathrm{NO}_{2} \mathrm{C}_{6} \mathrm{H}_{4} \mathrm{SH}$, (7) $p-\mathrm{MeC}_{6} \mathrm{H}_{4} \mathrm{SH}$, (8) 2-methyl-2-propanethiol, (9) pyrimidinethione, (10) $\alpha$-thioglycerol, (11) 2-mercaptobenzo-thiazole, (12) 4,4' thiobisbenzenethiol, (13) 2-aminoethanethiol hydrochloride, (14) PhSH. (e) Fluorescence intensity increase of TD-1 $(10 \mu \mathrm{M})$ incubating with thiophenol $(100 \mu \mathrm{M})$ during $3 \mathrm{~min}$. (f) Fluorescence intensity decrease of TD-1 $(10 \mu \mathrm{M})$ incubating with thiophenol $(100 \mu \mathrm{M})$ after 3-20 min due to probe seperation from solution.

To test the selectivity of TD-1 toward thiophenol and the possible interference from other analytes, the probe was incubated with a number of potential interfering species, aliphatic thiols and thiophenol derivatives. The fluorescence signal increased only in the presence of aromatic thiols (Fig. 1d). Other species were shown to not react with the probe and did not interfere with the sensing system when co-incubated with thiophenol (Fig. 1c).

The time-dependent fluorescence enhancement was measured by incubating thiophenol with TD-1 in PBS buffer. An incubation of excessive analyte was required to ensure that all TD-1 probes are transformed into fluorophore 4. After addition of thiophenol, the fluorescence intensity displayed a remarkable enhancement to maximum in 2-3 min (Fig. 1e). However, the fluorescence intensity was not stable and decreased slowly when stirring the solution for more than 3 min (Fig. 1f). We hypothesized that the poor water-solubility of compound $\mathbf{4}$ would lead to aggregated species that would separate from the solution, hence decreasing the fluorescence signal intensity. In our previous studies, TPE-based glycoclusters have excellent water solubility and similar structure as TD-1. For improving the water-solubility of TD-1, we self-assembled TPE-based glycoclusters $^{37}$ (TPE2S and TPE4S) and TD-1 to construct a glyco-probe, which was used to detect thiophenol (Scheme 1). We speculated that a better aqueous dispersibility could stabilize the fluorescence emission signal, allowing us to decrease the ratio of THF in PBS buffer.

\section{Spectral properties of glycol-probe}

To improve the water-solubility and fluorescence stability of TD-1, we self-assembled TPE2S with TD-1 and explored the fluorescence changes after incubating with thiophenol. The results indicated that TPE2S could enhance the fluorescence intensity probably due to an improvement of water solubility (Fig. 2a). Therefore, we compared the fluorescence enhancement efficiency of the two TPE-based glycoclusters (TPE2S and TPE4S) after self-assembly with TD-1 (Fig. 2b). The TPE2S assembly lead to a higher fluorescence intensity than that of the TPE4S assembly with probe TD-1, enabling thiophenol sensing in PBS aqueous buffer without the addition of THF. As a result, the TPE2S assembly was used for further investigation. To prove the assembly process, the molecular sizes of TPE2S, TD-1 and glyco-probe were measured by dynamic light scattering (DLS, Fig. 2c). TPE2S (blue) and TD-1 (green) in aggregated state displayed a large diameter $(200-300 \mathrm{~nm})$, which indicated that two molecules were in aggregated state. The size of the assembled glyco-probe (red) was smaller than TPE2S and similar to the size of TD-1. We supposed that the molecules of TD-1 were dispersed and inserted in the hydrophobic core of glyco-ball conjugating from several TPE2S, therefore the size of glyco-probe was similar to the aggregated TD-1. The optimal ratio between TPE2S and TD-1 was also investigated. A large excess of TPE2S was necessitated for achieving an optimal response to thiophenol with a 1:100 molar ratio of TD-1 to TPE2S (Fig. S7, ESI†).

Next, we investigated several basic fluorescence properties of the resulting glyco-probes for thiophenol detection. Compared with the AIE properties of TD-1, the glyco-probe displayed a better fluorescence increase with a varying PBS percentage of $50 \%-90 \%$ in THF. Importantly, the glycol-probe also displayed a satisfying emission intensity in complete PBS buffer (Fig. 2d). This result suggests that the glyco-probe with excellent watersolubility avoided the excessive intermolecular aggregation of 
compound $\mathbf{4}$, and hence was more suitable for thiophenol sensing in aqueous solution. Then, we compared the rate of reactivity of glyco-probe for thiophenol in PBS buffer (Fig. 2e) or a 95\% PBS buffer with 5\% THF (Fig. 2f). The glyco-probe displayed a rapidly increased fluorescence signal after incubating with thiophenol, and a stable fluorescence intensity when the probe responded completely over time. These results indicated that conjugation of TPE2S with TD-1 improved the water-solubility to prevent the probe precipitation. The longer response time (30 min) in PBS buffer in comparison to the mixture of PBS/THF (95:5) can be rationalized by the better solubility of thiophenol in THF, leading to a faster reaction time of 5 min.
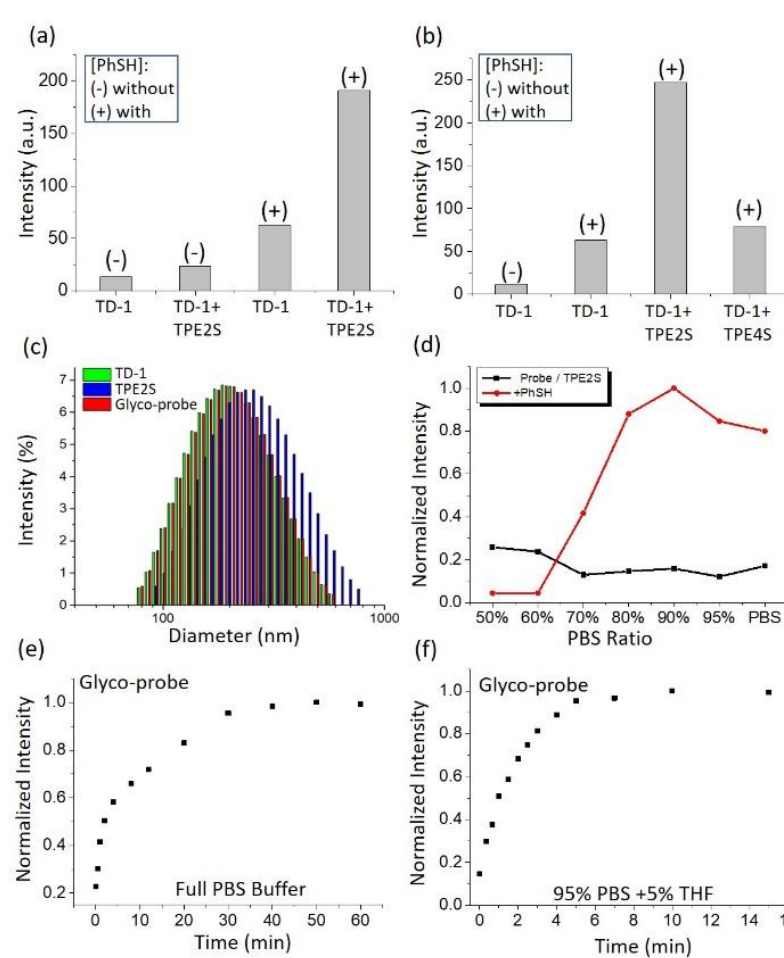

(f)

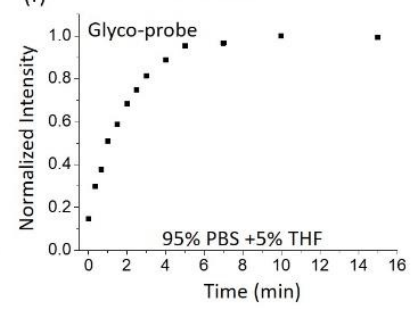

Figure 2 (a) Fluorescence changes of TD-1 $(10 \mu \mathrm{M})$ and glyco-probe after conjugation with TPE2S $(10 \mathrm{mM})$ improved the fluorescence intensity for thiophenol $(100 \mu \mathrm{M})$ detection in 95\% PBS (pH 7.3 $10 \mathrm{mM}$ ) with THF. (b) Fluorescence enhancement abilities of TPE2S and TPE4S $(10 \mathrm{mM})$ conjugating TD-1 $(10 \mu \mathrm{M})$ and detect thiophenol $(100 \mu \mathrm{M})$ in PBS buffer ( $\mathrm{pH} 7.3,10 \mathrm{mM}$ ). (c) DLS measurement of TD-1, TPE2S and glyco-probe. (d) AIE effect influenced the glyco-probe response to thiophenol $(100 \mu \mathrm{M})$ with PBS buffer $(\mathrm{pH} 7.3,10 \mathrm{mM})$ in THF during $5 \mathrm{~min}$. (e) and (f) Response time of glyco-probe to thiophenol $(100 \mu \mathrm{M})$ in PBS buffer or PBS buffer with $5 \%$ THF.

\section{PhSH Quantification}

To evaluate the sensitivity of the glyco-probe to thiophenol, the fluorescence signals at $560 \mathrm{~nm}$ of TD-1 and glyco-probe were measured with addition of thiophenol in PBS/THF (95:5) solution. TD-1 exhibited an unstable fluorescence intensity probably due to the poor water-solubility (Fig. 3a-b). In contrast, the glyco-probe showed a linear fluorescence enhancement with increasing thiophenol concentration (Fig. 3c). The linearity was verified from 0 to $14 \mu \mathrm{M}$ thus demonstrating that the glyco-probe could be used to detect thiophenol quantitatively (Fig. 3d). After adding more than $14 \mu \mathrm{M}$ thiophenol, the fluorescence intensity slowly reached a maximal emission intensity probably due to a complete conversion of the probes to compound 4 . The limit of detection (LOD) was calculated to be as low as $9.4 \mathrm{nM}$, which suggests that the probe could be applied for the determination of trace thiophenol in aqueous solution.
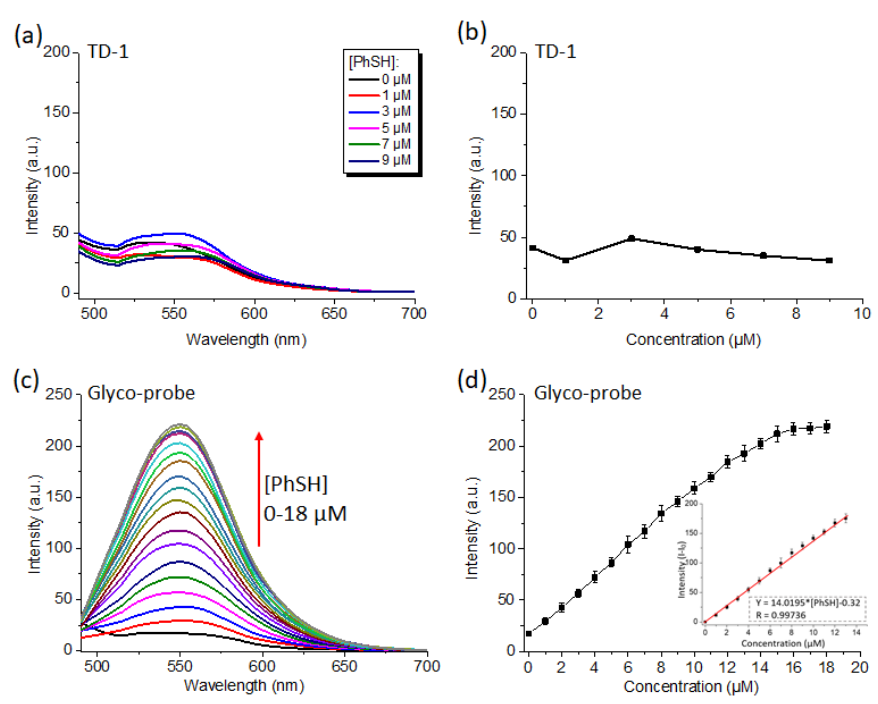

Figure 3 (a) and (b) Unstable fluorescence variation of TD-1 (10 $\mu \mathrm{M})$ response to thiophenol (0-9 $\mu \mathrm{M})$. (c) Fluorescence enhancement and (d) linear relationship of glycoprobe reponse to thiophenol $(0-18 \mu \mathrm{M})$ in $95 \%$ PBS buffer $(\mathrm{pH} 7.3,10 \mathrm{mM})$ with THF during $5 \mathrm{~min}$. $\left(\lambda_{\mathrm{ex}}=480 \mathrm{~nm}\right)$

Next, the glyco-probe was evaluated to quantify thiophenol in two real water samples from Saône River and Rhône River (Lyon, France). The glyco-probe provided homogenous fluorescence enhancements upon addition of thiophenol (0-3 $\mu \mathrm{M})$. The fluorescence increase (I-I $\left.\mathrm{I}_{0}\right)$ was similar to the results measured in PBS solution (Fig. S10, ESI $\dagger$ ). We have also injected exogenously known concentrations of thiophenol (0-3 $\mu \mathrm{M})$ into the real water samples (Table 1). The fluorescence signal recovery was always near $100 \%$ from the expected intensities through analysis by the titration curve. Therefore, the self-assembled glyco-probe between TPE2S and TD-1 could be used for thiophenol quantification in real water samples.

Table 1 Analysis of Thiophenol Concentrations in Real Water Samples

\begin{tabular}{cccc}
\hline $\begin{array}{c}\text { Sample } \\
\text { Saône River } \\
\text { water }\end{array}$ & PhSH spiked $(\mu \mathrm{M})$ & PhSH recovered $(\mu \mathrm{M})$ & Recovery $(\%)$ \\
& 1.00 & Not detect & 0 \\
& 2.00 & $0.97 \pm 0.09$ & 97 \\
\hline $\begin{array}{c}\text { Rhône River } \\
\text { water }\end{array}$ & 3.00 & $1.84 \pm 0.12$ & 92 \\
& 0 & $2.86 \pm 0.11$ & 95 \\
& 1.00 & Not detect & 0 \\
\hline
\end{tabular}

Conditions: The glyco-probe self-assembled from TD-1 $(10 \mu \mathrm{M})$ and TPE2S (10 mM) responded to thiophenol in $95 \%$ water samples with THF during $5 \mathrm{~min}$.

\section{Conclusion}

In a summary, detection of thiophenol is of interest as this toxic compound can pollute drinkable or recreative water. The design of a new fluorescent probe with a reactive 2,4-dinitrosulfonate 
moiety was achieved by the conjugation between a TPE AIEgen and a DCM core. The resulting probe could react with thiophenol to release a phenol group on the TPE-DCM moiety, thus enhancing the fluorescence emission at $570 \mathrm{~nm}$. Nevertheless, the poor water solubility of the resulting phenol did not allow stable measurements. We thus proposed a self-assembly strategy with water-soluble TPE-based glycoclusters, leading to a new glyco-probe which for the quantitative and selective detection of thiophenol in PBS solution as well as environmental water samples.

\section{Conflicts of interest}

There are no conflicts to declare.

\section{Acknowledgements}

The authors thank the financial support from the Natural Science Foundation of China (nos. 21788102, 91853201, 21722801 and 21776078), the Shanghai Municipal Science and Technology Major Project (No. 2018SHZDZX03) and the China Scholarship Council for a PhD stipend to L.D. (No. 201606740066).

\section{Notes and references}

1. A. Eychmüller and A. L. Rogach, Pure Appl. Chem., 2000, 72, 179188.

2. J. C. Love, L. A. Estroff, J. K. Kriebel, R. G. Nuzzo and G. M. Whitesides, Chem. Rev., 2005, 105, 1103-1170.

3. I. Rahman and W. MacNee, Am. J. Physiol. Lung Cell Mol. Physiol., 1999, 277, L1067-L1088.

4. (a) M. Zhang, Y. Wu, S. Zhang, H. Zhu, Q. Wu, L. Jiao and E. Hao Chem. Commun., 2012, 48, 8925-8927. (b) L. Yang, Y. Su, Y. Geng, Y. Zhang, X. Ren, L. He and X. Song, ACS Sens., 2018, 3, 1863-1869.

5. R. Munday, Free Radic. Biol. Med, 1989, 7, 659-673.

6. T. P. Hell and R. C. Lindsay, J. Environ. Sci. Heal. B, 1989, 24, 349360.

7. P. Amrolia, S. G. Sullivan, A. Stern and R. Munday, J. Appl. Toxicol., 1989, 9, 113-118

8. J.-X. He, T. Akao and T. Tani, Chem. Pharm. Bull., 2002, 50, 12331237.

9. T. Wang, E. Chamberlain, H. Shi, C. D. Adams and Y. Ma, Int. J. Environ. Anal. Chem., 2010, 90, 948-961.

10. X. Liu, F. Qi, Y. Su, W. Chen, L. Yang and X. Song, J. Mater. Chem. C, 2016, 4, 4320-4326.

11. X. Xie, M. Li, F. Tang, Y. Li, L. Zhang, X. Jiao, X. Wang and B. Tang, Anal. Chem., 2017, 89, 3015-3020.

12. G. Yin, T. Yu, T. Niu, P. Yin, H. Chen, Y. Zhang, H. Li and S. Yao, RSC Adv., 2017, 7, 46148-46154.

13. D. Kand, P. K. Mishra, T. Saha, M. Lahiri and P. Talukdar, Analyst 2012, 137, 3921-3924.

14. Y. Yue, F. Huo., Y. Zhang, J. Chao, R. Martínez-Máñez and C. Yin, Anal. Chem., 2016, 88, 10499-10503.

15. J. Li, C. F. Zhang, S. H. Yang, W. C. Yang and G. F. Yang, Anal. Chem., 2014, 86, 3037-3042.

16. S. Pagidi, N. K. Kalluvettukuzhy and P. Thilagar, Langmuir, 2018 34, 8170-8177.

17. D. Kand, P. S. Mandal, T. Saha and P. Talukdar, RSC Adv., 2014, 4, 59579-59586.

18. H. W. Liu, X. B. Zhang, J. Zhang, Q. Q. Wang, X. X. Hu, P. Wang and W. Tan, Anal. Chem., 2015, 87, 8896-8903.
19. L. Xiong, J. Ma, Y. Huang, Z. Wang and Z. Lu, ACS Sens., 2017, 2, 599-605.

20. (a) W. Jiang, Q. Fu, H. Fan, J. Ho and W. Wang, Angew. Chem. Int. Ed., 2007, 46, 8445-8448. (b) H. Guo, Y. Jing, X. Yuan, S. Ji, J. Zhao, X. Li and Y. Kan Org. Biomol. Chem., 2011, 9, 3844-3853.

21. D. G. Khandare, M. Banerjee, R. Gupta, N. Kumar, A. Ganguly, D. Singh and A. Chatterjee, RSC Adv., 2016, 6, 52790-52797.

22. D. Yu, Q. Zhai, S. Yang and G. Feng, Anal. Methods, 2015, 7, 75347539.

23. W. Jiang, Y. Cao, Y. Liu and W. Wang, Chem. Commun., 2010, 46, 1944-1946.

24. M. Zhang, T. Leng, Y. Shen and C. Wang, Analyst, 2018, 143, 756760.

25. Z. Guo, A. Shao and W.-H. Zhu, J. Mater. Chem. C, 2016, 4, 26402646.

26. X. Wang, Z. Gao, J. Zhu, Z. Gao and F. Wang, Polym. Chem., 2016 , 7, 5217-5220.

27. J. Mei, N. L. Leung, R. T. Kwok, J. W. Lam and B. Z. Tang, Chem. Rev., 2015, 115, 11718-11940.

28. J. Luo, Z. Xie, J. W. Lam, L. Cheng, H. Chen, C. Qiu, H. S. Kwok, X. Zhan, Y. Liu and D. Zhu, Chem. Commun. 2001, 1740-1741.

29. R. Hu, N. L. Leung and B. Z. Tang, Chem. Soc. Rev., 2014, 43, 44944562.

30. J. Li, N. Kwon, Y. Jeong, S. Lee, G. Kim and J. Yoon, ACS Appl. Mater. Interfaces, 2018, 10, 12150-12154.

31. H. Lin, H. Yang, S. Huang, F. Wang, D. M. Wang, B. Liu, Y. D. Tang and C. J. Zhang, ACS Appl. Mate.r Interfaces., 2018, 10, 1217312180.

32. W. T. Dou, Y. Zhang, Y. Lv, J. Wu, Y. Zang, C. Tan, J. Li, G. R. Chen and X. P. He, Chem. Commun., 2016, 52, 3821-3824.

33. D. K. Ji, Y. Zhang, Y. Zang, J. Li, G. R. Chen, X. P. He and H. Tian, Adv. Mater., 2016, 28, 9356-9363.

34. Y. Liu, D. K. Ji, L. Dong, N. Galanos, Y. Zang, J. Li, S. Vidal and X. P. He, Chem. Commun., 2017, 53, 11937-11940.

35. D. Jana, S. Boxi, P. P. Parui and B. K. Ghorai, Org. Biomol. Chem., 2015, 13, 10663-10674.

36. G. Zhao, Y. Zhu, S. Guang, F. Ke and H. Xu, New J. Chem., 2018 42, 555-563.

37. M. Donnier-Marechal, S. Abdullayev, M. Bauduin, Y. Pascal, M. Q Fu, X. P. He, E. Gillon, A. Imberty, E. Kipnis, R. Dessein and S. Vidal, Org. Biomol. Chem., 2018, 16, 8804-8809. 